\title{
Sensitivity Analysis of Shape Variables on Fire Extinguishing Nozzle for Reducing Flow Noise
}

\author{
Yo-Hwan Kim ${ }^{1}$, Jeong-Min Jin ${ }^{1}$, Yeong-Wan Je ${ }^{1}$ and Youn-Jea Kim ${ }^{2}$ \\ ${ }^{1}$ Graduate School of Mechanical Engineering, Sungkyunkwan University \\ Suwon 16419, Republic of Korea \\ kyh8030@skku.edu; wjdals3927@skku.edu \\ ${ }^{2}$ School of Mechanical Engineering, Sungkyunkwan University \\ Suwon 16419, Republic of Korea \\ yjkim@skku.edu
}

\section{Extended Abstract}

Sensitivity analysis (SA) is a study of how to determine relevance for uncertainties to various inputs, and it should be taken into account before selecting relevant variables to be investigated on how they correlate with one another [1]. Because of this, SA is usually used in a field of economics as a statistics method of evaluating the relative risk between investment targets [2]. Furthermore, as this is suitable to investigate the impact of many variables in engineering, in this study, design of experiment (DOE) technique, which is one of the SA methods [3], was applied to evaluate the effects of variables on a fire extinguishing nozzle using an inert gas. The objective function is selected to be the sound pressure level (SPL), and computational fluid dynamics (CFD) was used to obtain the reference data for the design of experiment. Furthermore, large eddy simulation (LES) method, which is well-known as an acceptable way to predict noise, was used for accurate numerical analysis [4]. The boundary conditions describe a situation where an inert gas(nitrogen) with 1.5MPa inlet pressure through the nozzle was discharged into an atmosphere $(1 \mathrm{~atm})$. All walls are adiabatic and no-slip conditions. The ultimate goal of this study is to reduce the sound pressure occurring from the fire extinguishing nozzle to avoid damage to data storage facility by minimizing the SPL [5]. Therefore, the sensitivity of the variables was evaluated from the numerically calculated velocity and pressure distributions and the results were graphically depicted. Results show that the change in the nozzle inner diameter was found to be the most sensitive factor compared to the others because it affects the mass flow rate. Although the change in the length of the outer guide was confirmed to be the second most sensitive, it has little effect on the mass flow rate.

\section{References}

[1] A. Saltelli, "Sensitivity Analysis for Importance Assessment," Risk Analysis., vol. 22, no. 3, pp. 579-590, 2002.

[2] C. Gourieroux, J. P. Laurent and O. Scaillet, "Sensitivity Analysis of Values at Risk," Journal of Empirical Finance., vol. 7, no. 3-4, pp. 225-245, 2000.

[3] J. P. Kleijnen, "Sensitivity Analysis and Optimization of System Dynamics Models: Regression Analysis and Statistical Design of Experiments," System Dynamics Review., vol. 11, no. 4, pp. 275-288, 1995.

[4] J. Nichols, S. Lele, P. Moin, F. Ham, G. Bres and J. Bridges, "Large-Eddy Simulation for Supersonic Rectangular Jet Noise Prediction: Effects of Chevrons," in 18th AIAA/CEAS Aeroacoustics Conference (33rd AIAA Aeroacoustics Conference), p. 2212, 2012.

[5] T. Dutta and A. R. Barnard, "Performance of Hard Disk Drives in High Noise Environments," Noise Control Engineering Journal., vol. 65, no. 5, pp. 386-395, 2017. 